\author{
Paola Sarchielli \\ Andrea Alberti \\ Beatrice Gallai \\ Francesca Coppola \\ Antonio Baldi \\ Ardesio Floridi \\ Virgilio Gallai
}

\section{Brain-derived neurotrophic factor in cerebrospinal fluid of patients with chronic daily headache: relationship with nerve growth factor and glutamate levels}

P. Sarchielli $(\varangle) \cdot$ A. Alberti • F. Coppola

A. Baldi • V. Gallai

Neuroscience Department,

University of Perugia,

Policlinico Monteluce,

Via E. Dal Pozzo, I-06126 Perugia, Italy

e-mail: neuro@netemedia.net

Tel.: +39-075-5783871

Fax: +39-075-5783583

B. Gallai

Department of Neurological and

Psychiatric Sciences in Childhood and

Adolescence,

La Sapienza University of Rome,

Rome, Italy

\section{A. Floridi}

Department of Internal Medicine,

Institute of Clinical and

Applied Biochemistry,

University of Perugia, Perugia, Italy

\begin{abstract}
Little has been done to investigate the biochemical basis of chronic daily headache $(\mathrm{CDH})$. Our group has recently demonstrated an increase in the cerebrospinal fluid (CSF) levels of nerve growth factor (NGF) in CDH patients, supporting the involvement of this growth factor in the abnormal processing of head pain in this pathological condition. Other members of the neurotrophin family, especially brain-derived neurotrophic factor (BDNF), have been hypothesized as being involved in the development of chronic head pain in patients affected by $\mathrm{CDH}$, but so far no data are available on this subject. BDNF, NGF and glutamate levels were determined in the CSF of 25 patients affected by $\mathrm{CDH}$ with a previous history of migraine. These levels were compared with those of a group of 20 control subjects, for whom the CSF examination and other instrumental investigations excluded diseases of the central and peripheral nervous systems.

Significantly higher levels of BDNF,
\end{abstract}

NGF and glutamate were found in $\mathrm{CDH}$ patients compared with control subjects $(p<0.0001, p<0.0002$ and $p<0.001$, respectively). A significant positive correlation emerged between CSF values of BDNF and those of $\operatorname{NGF}(\mathrm{r}=0.61, p<0.001)$ and glutamate $(\mathrm{r}=0.44, p<0.025)$ in $\mathrm{CDH}$ patients. No significant differences were detected in BDNF, NGF and glutamate levels between $\mathrm{CDH}$ patients with analgesic overuse and those without. These results support the involvement of BDNF in $\mathrm{CDH}$ through the potentiation of glutamatergic transmission involved in the processing of head pain. The significant correlation between BDNF and NGF levels suggests that NGF-mediated up-regulation of BDNF in central sites involved in long-term sensitization plays a key role in persistent head pain in $\mathrm{CDH}$ patients.

Key words Chronic daily headache • Central sensitization $\cdot$ Brain-derived neurotrophic factor $\cdot$ Nerve growth factor $\cdot$ Glutamate

\section{Introduction}

Neurotrophins are powerful modulators of both peripheral and central nervous system neurons, as well as regulators of long-term synaptic plasticity, including that which is believed to underlie central sensitization associated with chronic pain [1-5]. Brain-derived neurotrophic factor (BDNF) seems to play a crucial role in this process. This neurotrophin, which is synthesized by tyrosine kinase (trk)A-positive sensory neurons and acts through trkB receptors, is produced in experimental models of hyperalgesia [6-9]. Its role in sustained nociception is also supported by the finding of a blockage of inflammation-induced 
changes in sensory processing pathways by intrathecal trkBIgG fusion protein administration to sequester BDNF [10]. The synthesis of BDNF appears to be potentiated by nerve growth factor (NGF) which is up-regulated in hyperalgesia and pain models [11]. NGF treatment, in fact, dramatically increases BDNF levels in trkA nociceptive neurons where this neurotrophin is anterogradely transported, localized in dense vesicles and presumably released, at least at the spinal level [8, 12-14].

Potential targets of trkB-mediated modulation of central sensitization by BDNF are glutamate receptors. In particular, $\mathrm{N}$-methyl-D-aspartate (NMDA) receptors, which are strongly recruited in activated nociceptive pathways and mediate much of the polysynaptic C-fiber-evoked discharge, are activated by BDNF [15]. The trkB activation by BDNF leads to the phosphorylation of NMDA receptor subunits 1 and $2 \mathrm{~B}$ and increases the probability that these channels are in an open state $[16,17]$. This mechanism, clearly demonstrated in animal models of hyperalgesia and pain, is also believed to occur in patients with chronic daily headache $(\mathrm{CDH})$.

In a recent study we demonstrated a significant increase in the levels of NGF in the cerebrospinal fluid of patients with $\mathrm{CDH}$, and this increase appeared to be associated with an increase in the levels of the sensory neuropeptides calcitonin gene-related peptide (CGRP) and substance P (SP) [18]. On the basis of the evidence of the relationship between NGF and BDNF and also between BDNF and glutamate transmission in nociceptive processing in experimental models, we undertook the present study to investigate levels of BDNF in the cerebrospinal fluid (CSF) of patients with $\mathrm{CDH}$ (including patients with simple or combination analgesic abuse) and in the CSF of healthy age-matched controls. We also investigated possible correlations between the levels of BDNF, NGF and glutamate in $\mathrm{CDH}$ patient and control groups.

\section{Subjects and methods}

\section{Patients and controls}

A total of 25 consecutive patients affected by $\mathrm{CDH}$ and attending the Headache Center of the Neuroscience Department of the University of Perugia were included in the study. Inclusion criteria were: headache for at least 15 days per month for at least six months and the evolution of $\mathrm{CDH}$ from a previous history of migraine without aura. $\mathrm{CDH}$ was defined according to the criteria of Silberstein et al. [19]. Characteristics of headache attacks were defined according to the 1988 criteria of the International Headache Society (IHS) [20].

A control group was formed of 20 age- and sex-matched healthy subjects. None of the controls had a family history of migraine nor had they experienced episodic tension-type headache in the preceding 3 months. In these subjects we documented the absence of CNS disease (e.g. multiple sclerosis, vasculitis, other autoimmune CNS diseases) by CSF and blood examinations and, when necessary, by instrumental investigations including neuroimaging. We also documented the absence of systemic disease (e.g. diabetes, renal or hepatic dysfunction, inflammatory disease) by appropriate laboratory examinations.

Patients and controls were admitted to the Neurologic Clinic of the Neuroscience Department to undergo lumbar puncture for the determination of neuropeptide levels in CSF. All patients had headache at the time of lumbar puncture. No controls developed post-lumbar puncture headache.

The study protocol was approved by the Ethics Committee of the Municipality of Perugia. All study subjects gave written consent to participate in the study.

\section{Patient characterization}

Patients were subdivided into two groups on the basis of the intake of symptomatic drugs: normal or overuse. Following the criteria of Silberstein et al. [19], patients with analgesic overuse took $>1000$ mg acetylsalicylic acid (ASA) or acetaminophen for $>5$ days per week (simple analgesic overuse), or $>3$ tablets of any analgesic per day for $>3$ days per week (combination analgesic overuse).

Patients were given a diary in which to record on a daily basis, the occurrence, severity and duration of headache episodes for a 3 -month period. Intensity of head pain was scored on an ad hoc scale from 0 to 3 : 0 , no pain; 1 , pain not interfering with daily activities; 2 , moderate pain interfering with but not inhibiting daily activities; 3 , severe pain prohibiting daily activities. The recordings of the intensity pain of all headaches experienced by each patient in the 3-months using both the 4-point scale and 100 point VAS were averaged. In addition, the patients scored head pain on a visual analog scale (VAS) from 0 (no pain) to 100 (severe pain).

\section{Biochemical determinations}

Beta-NGF and BDNF were determined with a radioimmunoassay (RIA) method. Briefly, CSF samples were collected in tubes containing EDTA $(1 \mathrm{mg} / \mathrm{ml})$ and kallikrein $(500 \mathrm{IU} / \mathrm{ml})$. After centrifugation at $3000 \mathrm{rpm}$ for $15 \mathrm{~min}$ at $4^{\circ} \mathrm{C}, \mathrm{CSF}$ samples were collected and stored at $-80^{\circ} \mathrm{C}$. At the time of analysis, they were thawed and immediately acidified with an equal volume of $0.1 \%$ trifluoroacetic acid. The samples were applied to a SEP-C18 column and BDNF and NGF were eluted from the mixture with $60 \%$ acetonitrile in $0.1 \%$ trifluoroacetic acid. The eluate was evaporated to dryness in a centrifugal concentrator (Supervap PL-CC-180) and dissolved in RIA buffer. The amounts of NGF and BDNF in the samples were determined by comparison to standard curves made from serial 4fold dilutions of pure NGF and BDNF in RIA buffer.

Glutamate levels in CSF were measured by HPLC, using ophthalaldehyde precolumn derivatization and electrochemical plus fluorometric detection. A Bio-Rad pump apparatus coupled to a Coulochem 5100A electrochemical detector (ESA, Chelmsford MA, USA) with a coulometric analytic cell (Model 5011) and a FP-920 fluorescence detector (Jasco International) was used with a C18 Rosil HL (150×4.6 mm internal diameter, Bio-Rad 
Laboratories) reversed-phase column. Phosphate buffer, prepared from $25 \mathrm{mmol} / \mathrm{l}$ potassium phosphate and $150 \mathrm{ml} / \mathrm{l}$ acetonitrile, was applied as the mobile phase at a flow rate of $1.0 \mathrm{ml} / \mathrm{min}$. The glutamate content was evaluated after comparison with an external glutamate calibration solution.

\section{Statistical analysis}

ANOVA with Tukey test was used to compare the mean NGF and BDNF values between $\mathrm{CDH}$ patients and control subjects, and between $\mathrm{CDH}$ patients with analgesic overuse and those without. Fisher's least significance difference (LSD) was used to compare the main effect means in ANOVA. Five percent for two-sided tests was chosen as a minimum level of statistical significance.

Pearson's coefficient was calculated to determine possible correlations among BDNF, NGF and glutamate levels in patient and control groups. Correlations between BDNF and age (years), duration of disease (years), number of headache days per month, and headache intensity scores were also examined in the $\mathrm{CDH}$ patient group.

\section{Results}

We studied possible correlations between the neuropeptides NGF and BDNF and headache intensity in 25 patients with $\mathrm{CDH}$ and in 20 healthy controls (Table 1).
According to Silberstein et al.'s criteria for CDH forms [19], all 25 patients were classified as having transformed migraine. When we applied the 1988 IHS criteria [20], 10 of 25 patients were classified as having migraine without aura. The remaining 15 patients were considered to have migraine without aura not fulfilling IHS criteria, because their headache was essentially continuous $(n=5)$, they lacked the mandatory number of associated symptoms $(n=7)$ or a pain factor was missing $(n=3)$.

Analgesic overuse was found in 15 of the 25 patients. A total of 4 patients used more than 1 simple analgesic while 11 used both simple and combination analgesics. None used ergot derivatives or drugs containing codeine, nor did they take analgesics for other painful conditions. The mean monthly drug intake was $72.4(\mathrm{SD}=16.4)$ tablets or suppositories for the $15 \mathrm{CDH}$ patients with analgesic overuse.

Patients with $\mathrm{CDH}$ showed significantly higher BDNF and NGF levels compared to control subjects (Table 2). No significant difference in BDNF or NGF values emerged between $\mathrm{CDH}$ patients with and without analgesic overuse. Significantly higher values of glutamate were found in the CSF of CDH patients when compared to control subjects, but there was no significant difference between $\mathrm{CDH}$ patients with and without analgesic overuse.

A significant positive correlation emerged between BDNF and NGF values in the CSF of CDH patients (Fig. 1), but not in control subjects. CSF levels of BDNF were significantly correlated with $\mathrm{CSF}$ glutamate levels in $\mathrm{CDH}$

Table 1 Characteristics of patients with chronic daily headache (CDH) and control subjects. Values are mean (SD) unless otherwise indicated

\begin{tabular}{lcc}
\hline & CDH patients $(\mathrm{n}=25)$ & Control subjects $(\mathrm{n}=20)$ \\
\hline Men, $\mathrm{n}(\%)$ & $7(28)$ & $7(35)$ \\
Age, years & $46.5(8.4)$ & $44.8(9.1)$ \\
CDH duration, years & $13.1(4.6)$ & NA \\
Analgesic overuse, $\mathrm{n}(\%)$ & $15(60)$ & NA \\
Headache days/month, $\mathrm{n}$ & $23.1(4.9)$ & NA \\
Headache intensity & $2.1(1.0)$ & NA \\
$\quad$ Scale from 0 to 3 & $74.4(11.6)$ & \\
$\quad$ VAS from 0 to 100 & & NA \\
\hline
\end{tabular}

$V A S$, visual analog scale

Table 2 BDNF, NGF and glutamate levels in CSF in CDH patients and in controls. Values are mean (SD)

\begin{tabular}{lcccc}
\hline & \multirow{2}{*}{$\begin{array}{c}\text { Controls } \\
(\mathrm{n}=20)\end{array}$} & \multicolumn{2}{c}{ CDH patients } \\
\cline { 3 - 5 } & & All $(\mathrm{n}=25)$ & Analgesic overuse $(\mathrm{n}=15)$ & No analgesic overuse $(\mathrm{n}=10)$ \\
\hline $\mathrm{BDNF}, \mathrm{pg} / \mathrm{ml}$ & $14.3(3.4)$ & $33.8(5.4)^{*}$ & $33.0(8.9)$ & $32.8(4.9)$ \\
$\mathrm{NGF}, \mathrm{pg} / \mathrm{ml}$ & $11.7(2.7)$ & $39.8(5.8)^{* *}$ & $39.4(6.8)$ & $38.33(5.27)$ \\
Glutamate, $\mu \mathrm{mol} / \mathrm{l}$ & $1.4(0.3)$ & $2.7(0.50)$ & $2.2(0.4)$ & $2.2(0.4)$ \\
\hline
\end{tabular}

$* p<0.0001,{ }^{* *} p<0.0005$ vs. controls 


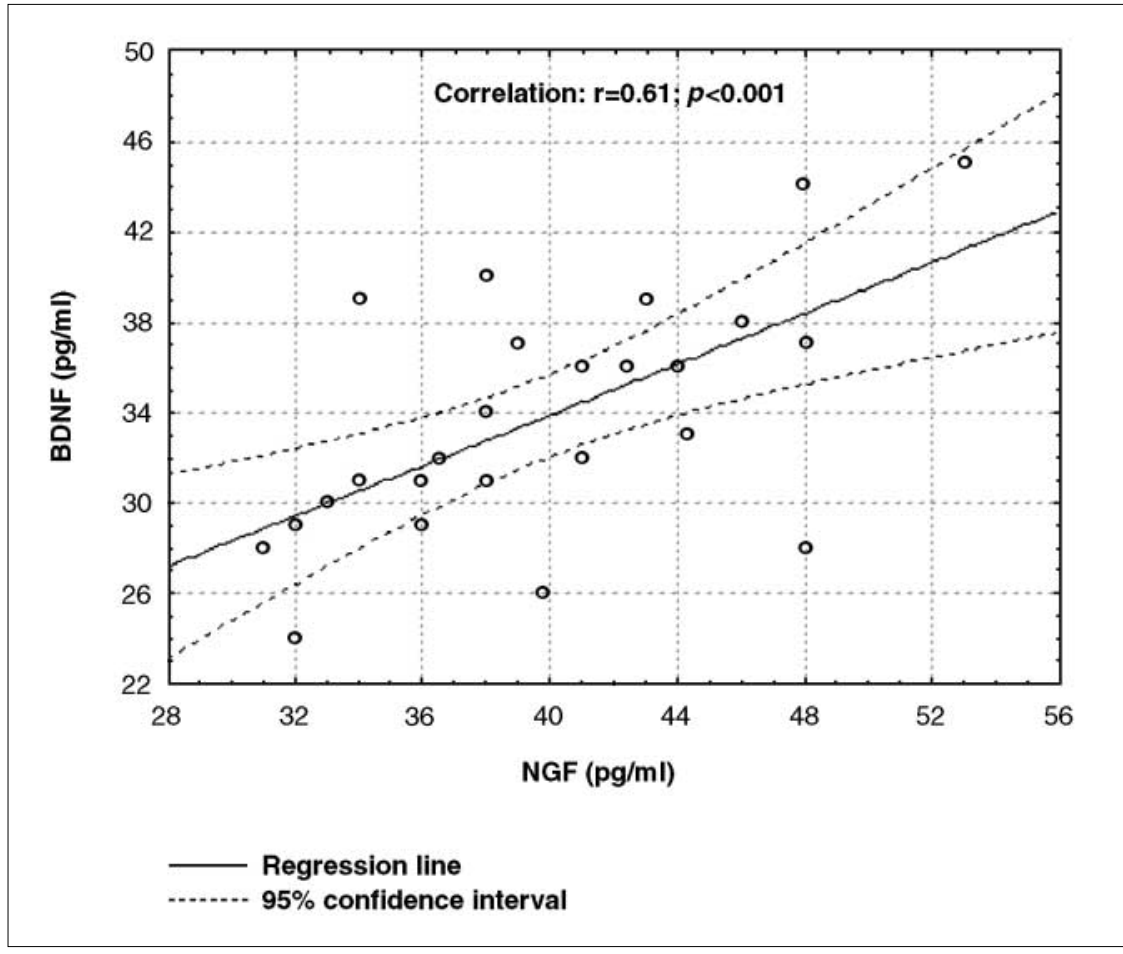

Fig. 1 Correlation between BDNF and NGF levels in CSF of CDH patients

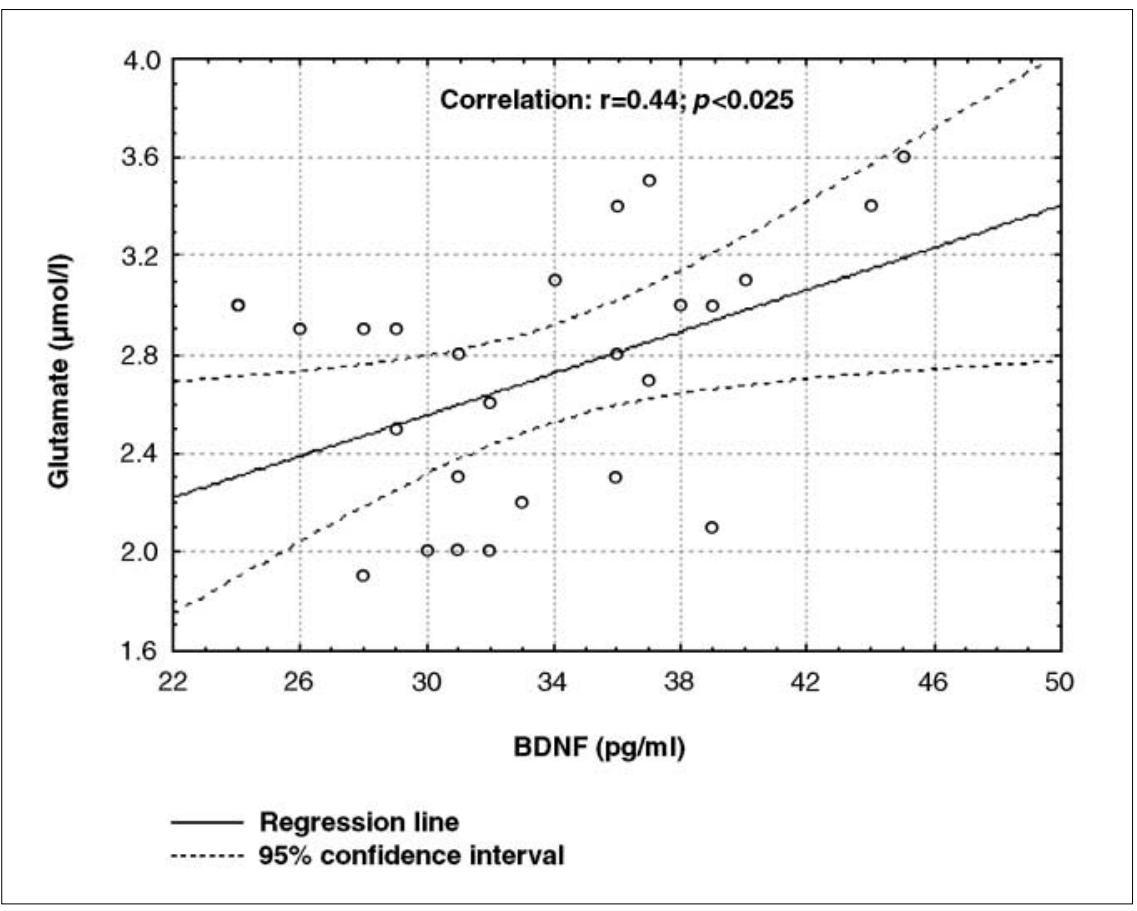
mate levels in $\mathrm{CSF}$ of $\mathrm{CDH}$ patients

patients (Fig. 2), but this correlation was not evident in controls. Due to this correlation, a statistically significant $r$ value was also found between NGF and glutamate levels in the CSF of $\mathrm{CDH}$ patients $(\mathrm{r}=0.42 ; p<0.035)$.
CSF levels of NGF and to a lesser extent BDNF correlated significantly with the duration (in years) of chronic headache and the number of headache days per month, but not with VAS scores (Table 3) or pain intensity (not shown). 
Table 3 Correlation of BDNF, NGF and glutamate levels in CSF with clinical headache parameters in 25 patients with CDH

\begin{tabular}{lccc}
\hline & CDH duration & Headache days/month & VAS score \\
\hline BDNF & $\mathrm{r}=0.31(p=\mathrm{ns})$ & $\mathrm{r}=0.38(p=0.05)$ & $\mathrm{r}=0.25(p=\mathrm{ns})$ \\
NGF & $\mathrm{r}=0.40(p<0.0005)$ & $\mathrm{r}=0.56(p<0.002)$ & $\mathrm{r}=0.20(p=\mathrm{ns})$ \\
Glutamate & $\mathrm{r}=0.23(p=\mathrm{ns})$ & $\mathrm{r}=0.39(p=\mathrm{ns})$ & $\mathrm{r}=0.11(p=\mathrm{ns})$ \\
\hline
\end{tabular}

$n s$, not significant

\section{Discussion}

A key mechanism that contributes to many persistent pain states is the increased excitability and firing of sensory pathways in the spinal cord and supraspinal sites involved in nociception. This phenomenon, known as central sensitization, has been suggested to be involved in the generation of chronic head pain. The biochemical basis of this process in patients affected by $\mathrm{CDH}$ has not been elucidated. In particular, little has been done to investigate modifications in some neurotransmitters and neuromodulators in the CSF, which could certainly provide clear information about biochemical changes occurring at the central level in these patients.

In the present study we have demonstrated an increase in CSF levels of BDNF in CDH patients, substantiating the participation of this neurotrophin in the induction and maintenance of chronic head pain. In this regard we should point out the role played by BDNF in central sensitization in animal models of induced pain, where an up-regulation of BDNF and an increased expression of trkB have been shown in sensitized $C$ nociceptors $[15,21]$. The increased production of BDNF revealed in the CSF of $\mathrm{CDH}$ patients examined also suggests the occurrence of BDNF up-regulation in this human chronic pain condition.

The present study also confirmed our previous finding of significantly higher levels of NGF in the CSF of CDH patients [18]. A significant positive correlation emerged between the CSF levels of BDNF and NGF in CDH patients. This suggests that BDNF is produced in $\mathrm{CDH}$ in an NGFdependent manner, as in experimental models of pain. The nociceptive effect of NGF, an increase in which is found in basal and stimulus-induced hyperalgesia, seems to be exerted through the up-regulation of BDNF mRNA and protein, and thus leads to increased sensitivity and action potentials in nociceptive neurons [22, 23]. This mechanism, clearly shown in animal pain models, could also occur in $\mathrm{CDH}$ and sustain central sensitization as the basis of chronic head pain in affected patients.

An increase of glutamate transmission has been demonstrated to underlie central sensitization and chronic pain in experimental animal models. The demonstration of increased glutamate levels in the $\mathrm{CSF}$ of $\mathrm{CDH}$ patients examined in the present research is entirely compatible with experimental evidence linking central sensitization to NMDA-mediated nociceptive responses and concurs with recent data from our group [24].

The neurotrophin NGF may be an endogenous modulator of synaptic activity via enhancement of NMDA-mediated responses [25]. In particular, BDNF is responsible for the induction of NRB2 subunit expression, which defines the pharmacological and biophysical properties of these receptors and also induces phosphorylation of the NR1 and NR2B subunits [26]. By means of these changes, BDNF is able to enhance an NMDA response via a threefold increase in NMDA receptor open time [27, 28]. The relationship between BDNF and glutamate levels in the CSF of our CDH patients also suggests a direct relationship between the increase of NGF and the release of the excitatory amino acid glutamate. This is in agreement with experimental evidence supporting the enhancement of a spontaneous release of glutamate in primary neural cultures and suggesting that the role of BDNF functions as a retrograde modulator of presynaptic transmitter release.

On the other hand, there are other experimental findings that deny the intervention of this mechanism, at least within the spinal cord. This is because not all subpopulations of sensory neurons expressing BDNF display trkB receptors and therefore are probably insensitive to this neurotrophin [29]. No data in this regard are available for nociceptive neurons of the trigeminal nucleus caudalis.

Other putative, even indirect effects of BDNF could account for an increased activity of the glutamatergic pathway. The activation of NMDA by BDNF could be responsible for the appearance of neural nitric oxide synthase (NOS) in nociceptors in spinal and supraspinal sites involved in pain processing $[30,31]$. Diffusible NO produced as a consequence of the increased NOS activity could account for the enhancement of glutamate release and contribute therefore to the potentiation of NMDA receptor activity. Glutamate can stimulate BDNF release; therefore, the association between these two factors is more complex, and needs to be further clarified [32].

In the present research, the abuse of simple or combination analgesics seems not to have influenced the biochemical changes investigated, which appear to be more strictly 
related to the duration of $\mathrm{CDH}$ and to the number of headache days per month, and therefore to the chronic pain state, particularly for NGF and BDNF.

The most consistent concept which could be summarized from the present and previous results is the association between NGF and BDNF levels in CDH. BDNF could be responsible for increased glutamatergic transmission, and NGF is strongly associated with sensory neuropeptide release as suggested by a previous study of our group [18]. All of these biochemical changes could underlie central sensitization, which may be involved in headache chronicity.
The effects of a therapeutic approach, especially prophylactic intervention on the biochemical changes evidenced in the present study in CDH patients, should be investigated in future research.

The results obtained in our group of $\mathrm{CDH}$ patients, however, cannot be considered at the moment to be specific for $\mathrm{CDH}$. Control patient groups with chronic pain from other causes should be investigated to verify if the findings of the present study are specific for $\mathrm{CDH}$ or common to other chronic painful conditions. Thus, they could be more indicative of chronic pain per se and not of chronic head pain.

\section{References}

1. Lu B, Chow A (1999) Neurotrophins and hippocampal synaptic transmission and plasticity. J Neurosci Res 58(1):76-87

2. Thompson SW, Bennett DL, Kerr BJ, Bradbury EJ, McMahon SB (1999) Brain-derived neurotrophic factor is an endogenous modulator of nociceptive responses in the spinal cord. Proc Natl Acad Sci USA 96(14):7714-7718

3. Mendell LM, Munson JB, Arvanian VL (2001) Neurotrophins and synaptic plasticity in the mammalian spinal cord. J Physiol 533(Pt 1):91-97

4. Heppenstall PA, Lewin GR (2001) BDNF but not NT-4 is required for normal flexion reflex plasticity and function. Proc Natl Acad Sci USA 98(14):8107-8112

5. Woolf CJ, Salter MW (2000) Neuronal plasticity: increasing the gain in pain. Science 288(5472):1765-1769

6. Barbacid M (1994) The TrK family of neurotrophin receptors. J Neurobiol 25(11):1386-1403

7. Yan Q, Rosenfeld RD, Matheson CR, Hawkins N, Lopez OT, Bennett L, Welcher AA (1997) Expression of brain-derived neurotrophic factor protein in the adult rat central nervous system. Neuroscience 78(2):431-448

8. Zhou XF, Rush RA (1996)

Endogenous brain-derived neurotrophic factor is anterogradely transported in primary sensory neurons. Neuroscience 74(4):945-953

9. Aloyz R, Fawcett JP, Kaplan DR, Murphy RA, Miller FD (1999) Activity-dependent activation of TrkB neurotrophin receptors in the adult CNS. Learn Mem 6(3):216-231
10. McAllister AK, Katz LC, Lo DC (1997) Opposing roles for endogenous BDNF and NT-3 in regulating cortical dendritic growth. Neuron 18(5):767-778

11. Lewin GR, Rueff A, Mendell LM (1994) Peripheral and central mechanisms of NGF-induced hyperalgesia. Eur J Neurosci 6(12):1903-1912

12. Woolf CJ, Safieh-Garabedian B, Ma QP, Crilly P, Winter J (1994) Nerve growth factor contributes to the generation of inflammatory sensory hypersensitivity. Neuroscience 62(2):327-331

13. Michael GJ, Averill S, Nitkunan A, Rattray M, Bennett DL, Yan Q, Priestley JV (1997) Nerve growth factor treatment increases brain-derived neurotrophic factor selectively in TrkA-expressing dorsal root ganglion cells and in their central terminations within the spinal cord. J Neurosci 17(21):8476-8490

14. Cho HJ, Kim JK, Zhou XF, Rush RA (1997) Increased brain-derived neurotrophic factor immunoreactivity in rat dorsal root ganglia and spinal cord following peripheral inflammation. Brain Res 764 (1-2):269-272

15. Kerr BJ, Bradbury EJ, Bennett DL, Trivedi PM, Dassan P, French J, Shelton DB, McMahon SB, Thompson SW (1999) Brain-derived neurotrophic factor modulates nociceptive sensory inputs and NMDA-evoked responses in the rat spinal cord. J Neurosci 19(12):5138-5148
16. Small DL, Murray CL, Mealing GA, Poulter MO, Buchan AM, Morley P (1998) Brain derived neurotrophic factor induction of N-methyl-D-aspartate receptor subunit NR2A expression in cultured rat cortical neurons. Neurosci Lett 252(3):211-214

17. Suen PC, Wu K, Levine ES, Mount HT, Xu JL, Lin SY, Black IB (1997) Brain-derived neurotrophic factor rapidly enhances phosphorylation of the postsynaptic N-methyl-D-aspartate receptor subunit 1. Proc Natl Acad Sci USA 94(15):8191-8195

18. Sarchielli P, Alberti A, Floridi A, Gallai V (2001) Levels of nerve growth factor in cerebrospinal fluid of chronic daily headache patients. Neurology 57(1):132-134

19. Silberstein SD, Lipton RB, Solomon S, Mathew NT (1994) Classification of daily and near- daily headaches: proposed revisions to the IHS criteria. Headache 34(1):1-7

20. Headache Classification Committee of the International Headache Society (1988) Classification and diagnostic criteria of headache disorders, cranial neuralgias and facial pain. Cephalalgia 8[Suppl 7]:1-96

21. Mendell LM. Albers KM, Davis BM (1999) Neurotrophins, nociceptors, and pain. Microsc Res Tech 45(4-5):252-261

22. Apfel SC, Wright DE, Wiideman AM, Dormia C, Snider WD, Kessler JA (1996) Nerve growth factor regulates the expression of brain-derived neurotrophic factor mRNA in the peripheral nervous system. Mol Cell Neurosci 7(2):134-142 
23. Mannion RJ, Costigan M, Decosterd I, Amaya F, Ma QP, Holstege JC, Ji RR, Acheson A, Lindsay RM, Wilkinson GA, Woolf CJ (1999) Neurotrophins: peripherally and centrally acting modulators of tactile stimulus-induced inflammatory pain hypersensitivity. Proc Natl Acad Sci USA 96(16):9385-9390

24. Gallai V, Alberti A, Coppola F, Floridi A, Sarchielli P (2002) Glutamate and nitric oxide pathway in chronic daily headache: evidence from cerebrospinal fluid. Cephalalgia (in press)

25. Li YX, Zhang Y, Lester HA, Schuman EM, Davidson N (1998) Enhancement of neurotransmitter release induced by brain-derived neurotrophic factor in cultured hippocampal neurons. J Neurosci 18(24):10231-10240
26. Lin SY, Wu K, Levine ES, Mount HT, Suen PC, Black IB (1998) BDNF acutely increases tyrosine phosphorylation of the NMDA receptor subunit 2B in cortical and hippocampal postsynaptic densities. Brain Res Mol Brain Res 55(1):20-27

27. Levine ES, Crozier RA, Black IB, Plummer MR (1998) Brain-derived neurotrophic factor modulates hippocampal synaptic transmission by increasing $\mathrm{N}$-methyl-D-aspartic acid receptor activity. Proc Natl Acad Sci USA 95(17):10235-10239

28. Jarvis CR, Xiong ZG, Plant JR, Churchill D, Lu WY, MacVicar BA, MacDonald JF (1997) Neurotrophin modulation of NMDA receptors in cultured murine and isolated rat neurons. J Neurophysiol 78(5):2363-2371
29. McMahon SB, Armanini MP, Ling LH, Phillips HS (1999) Expression and coexpression of Trk receptors in subpopulations of adult primary sensory neurons projecting to identified peripheral targets. Neuron 12:1161-1171

30. Huber KA, Krieglstein K, Unsicker K (1995) The neurotrophins BDNF, NT-3 and -4 , but not NGF, TGF-beta1 and GDNF, increase the number of NADPH-diaphorase-reactive neurons in rat spinal cord cultures. Neuroscience 69(3):771-779

31. Bennett DL, French J, Priestley JV, McMahon SB (1996) The effects of BDNF on cfos and NOS expression in dorsal horn neurons of the spinal cord. Soc Neurosci 22:396

32. Hartmann M, Heumann R, Lessmann V (2001) Synaptic secretion of BDNF after high-frequency stimulation of glutamatergic synapses. EMBO J 20(21):5887-5897 